\title{
FITOTERÁPICOS NA ATENÇÃO BÁSICA À SAÚDE: UMA EXPERIÊNCIA NA REGIÃO SUL DO BRASIL
}

\section{PHYTOTHERAPEUTICS IN BASIC HEALTH CARE: AN EXPERIENCE IN THE SOUTH REGION OF BRAZIL}

\author{
Caroline Gribner ${ }^{1 \star}$, Yanna Dantas Rattmann², Eliane Carneiro Gomes ${ }^{3}$
}

1 - Doutoranda do Programa de Pós-Graduação em Ciências Farmacêuticas - Universidade Federal do Paraná (UFPR) - Curitiba/PR- Brasil.

2- Universidade Federal do Paraná (UFPR), Programa de Pós-graduação em Saúde Coletiva, Professora Adjunta do Departamento de Saúde Comunitária. Curitiba/Paraná - Brasil.

3 - Universidade Federal do Paraná (UFPR), Programa de Pós-graduação em Ciências Farmacêuticas, Programa de Pós-graduação em Saúde Coletiva, Professora Associada do Departamento de Saúde Comunitária. Curitiba/PR- Brasil.

*Autor para correspondência: carol_gribner@yahoo.com.br

\section{RESUMO:}

O uso da fitoterapia e plantas medicinais na terapia humana é uma das práticas terapêuticas mais antigas da humanidade, apresentando origem tanto do conhecimento popular como das experiências científicas. Os fitoterápicos constam na Relação Nacional de Medicamentos Essenciais compondo o grupo do componente básico da assistência farmacêutica estando disponíveis para aquisição e dispensação aos usuários do Sistema Único de Saúde. Neste contexto, buscou-se verificar a utilização de Fitoterápicos Industrializados na Atenção Básica à Saúde nos municípios da Região Metropolitana de Curitiba, a fim de avaliar quais possuem fitoterápicos industrializados como alternativa terapêutica, bem como quais são disponibilizados pelos municípios. Os dados foram obtidos por meio de consulta à Relação Municipal de Medicamentos Essenciais, além de informações provenientes das secretarias municipais de saúde de cada município que compõem a Região Metropolitana de Curitiba. Dos 29 municípios da Região Metropolitana de Curitiba, 23 dispensam fitoterápicos industrializados, sendo que 6 municípios optam por não disponibilizá-los. Entre os fitoterápicos dispensados, 4 deles se mostram mais frequentes. São eles: Xarope de Guaco (87\%), Isoflavona de Soja (48\%), Ginkgo biloba $(30 \%)$ e Espinheira Santa (22\%). Quando avaliada a diversidade dos fitoterápicos, observou-se que, em média, os municípios possuem em seu elenco 1 ou 3 fitoterápicos industrializados. Conclui-se que a maioria dos municípios da Região Metropolitana de Curitiba opta por utilizar fitoterápicos como alternativa de tratamento, e que, embora ocorram várias ações no sentido de implementar os fitoterápicos no Sistema Único de Saúde, esse crescimento ainda não foi suficiente para torná-los uma unanimidade nos serviços de saúde.

Palavras-chave: Fitoterápicos, Sistema Único de Saúde, Práticas Integrativas.

\begin{abstract}
:
The use of phytotherapy and medicinal plants in human therapy is one of the oldest therapeutic practices of mankind, presenting origin of both popular knowledge and scientific experiments. The phytotherapics are listed in the National List of Essential Medicines as the basic component of pharmaceutical care and are available for purchase and dispensation to the users of the Unified Health System. In this context, we sought to verify the use of
\end{abstract}


Industrialized Herbal Medicines in Basic Health Care in municipalities of the Metropolitan Region of Curitiba, in order to evaluate which have industrialized herbal medicines as a therapeutic alternative, as well as which are available by the municipalities. The data were obtained through consultation with the Municipal Association of Essential Medicines, in addition to information from the municipal health secretariats of each municipality that compose the Metropolitan Region of Curitiba. Of the 29 municipalities in the Metropolitan Region of Curitiba, 23 dispensed industrialized herbal medicines, and 6 municipalities choose not to make them available. Among the phytotherapics dispensed, 4 of them are more frequent. They are: Guaco Syrup (87\%), Soy Isoflavone (48\%), Ginkgo biloba (30\%) and Espinheira Santa (22\%). When evaluating the diversity of phytotherapics, it was observed that, on average, the municipalities have in their list 1 or 3 industrialized herbal medicines. It is concluded that the majority of municipalities in the Metropolitan Region of Curitiba choose to use phytotherapics as an alternative treatment, and that although there are several actions to implement herbal medicines in the Unified Health System, health services. Key words: Drug Phytotherapic, Unified Health System, Integrative Practices.

\section{INTRODUÇÃO}

O uso da fitoterapia e plantas medicinais na terapia humana é uma das práticas terapêuticas mais antigas da humanidade, apresentando origem tanto do conhecimento popular como das experiências científicas (FERREIRA et al., 2014).

A Fitoterapia segundo a legislação brasileira é definida como um recurso terapêutico que se caracteriza pelo uso de plantas medicinais em suas diferentes formas farmacêuticas, não utilizando substâncias ativas isoladas, ainda que possuam origem vegetal (BRASIL, 2006a).

Neste contexto temos os medicamentos fitoterápicos, os quais são produtos industrializados tecnicamente elaborados, originados de modo exclusivo de matériasprimas vegetais, que de forma complementar pode ser caracterizado pelo conhecimento da sua eficácia e eficiência baseada por meio de evidências clínicas, riscos relacionados ao seu uso, capacidade de reprodutibilidade e manutenção de sua qualidade (BRASIL, 2006b; BRASIL, 2012a; NIERO et al., 2003; BRASIL, 2014).

Em relação a sua segurança e eficácia, a comprovação pode ser realizada por meio de ensaios não clínicos e clínicos; ou por registro simplificado comprovado pela sua presença na Lista de medicamentos fitoterápicos de registro simplificado, de acordo com a Instrução Normativa-IN n 2, de 13 de maio de 2014; pela presença nas monografias de fitoterápicos de uso bem estabelecido da Comunidade Europeia (BRASIL, 2014).

Recentemente foi estabelecida a definição de produto tradicional fitoterápico, que compreende aqueles obtidos exclusivamente de matérias-primas vegetais, cuja segurança e efetividade são baseadas em dados da literatura técnico-científica através da 
comprovação de uso seguro e efetivo para um período mínimo de 30 anos, que incluem publicações nacionais e internacionais; ou registro simplificado, que deverá ser comprovado pela presença na Lista de produtos tradicionais fitoterápicos de registro simplificado, conforme IN n² 2, de 13 de maio de 2014; ou presença nas monografias de fitoterápicos de uso tradicional da Comunidade Europeia. Não sendo necessárias evidências clínicas e acompanhamento médico para sua utilização (BRASIL, 2014).

Ambas as definições não são válidas para aqueles que possuam em sua composição substâncias ativas isoladas, de qualquer origem, sintética ou natural, nem as associações destas com extratos vegetais (BRASIL, 2006b).

A utilização dos fitoterápicos como um recurso terapêutico foi oficialmente reconhecida pela Organização Mundial da Saúde em 1978. De forma conjunta, houve um fortalecimento e crescimento do interesse popular em implantar a fitoterapia no Sistema Único de Saúde (SUS), onde no decorrer da década de 90 e após, diversas Resoluções, Portarias e Relatórios foram elaborados tendo como foco a disponibilização de medicamentos fitoterápicos em unidades de saúde (BRASIL, 2001).

No Brasil a utilização de fitoterápicos e plantas medicinais é reconhecida oficialmente por meio do Decreto no 5.813, de 22 de junho de 2006, que aprovou o Programa Nacional de Plantas Medicinais e Fitoterápicos. Corroborando com este programa, ocorreu a inserção dos fitoterápicos no SUS por meio da Portaria n 971, de 03 de Maio de 2006, que aprovou a Política Nacional de Práticas Integrativas e Complementares no SUS, onde se encontram as diretrizes para a implementação das Plantas Medicinais e Fitoterapia (BRASIL, 2006a; BRASIL, 2006b).

Desta maneira, diversos investimentos foram realizados, com a finalidade de auxiliar a implantação de programas e projetos que possibilitem a produção e distribuição de plantas e fitoterápicos, para que se tenha a fitoterapia como uma opção de ampliar a terapêutica oferecida (BRASIL, 2012b).

Assim, é de fundamental importância à inserção da fitoterapia nas políticas de saúde presentes em municípios e estados, com garantia de segurança, eficácia, qualidade e reprodutibilidade do efeito; tendo em vista a melhoria da atenção primária à saúde e a inclusão social, para estar de acordo com os princípios que estruturam o SUS; a universalidade, integralidade e equidade (BRASIL, 2006b; BRASIL, 2012a; BRASIL, 1990).

Diante desta abordagem, este estudo pretende avaliar a inserção da fitoterapia na atenção básica à saúde nos municípios da Região Metropolitana de Curitiba (RMC), composta por 29 municípios, por meio da disponibilização de fitoterápicos industrializados 
em suas Unidades Básicas de Saúde. Além disso, pretende-se realizar um levantamento dos fitoterápicos industrializados dispensados aos usuários.

\section{MATERIAL E MÉTODOS}

Trata-se de um estudo exploratório, com abordagem quantitativa realizada no período de setembro a novembro de 2015. Primeiramente se buscou conhecer a composição de municípios da RMC, por meio de pesquisa ao Instituto Brasileiro de Geografia e Estatística.

Para obter a relação dos municípios da RMC que distribuem fitoterápicos industrializados na atenção básica à saúde, foi realizada uma consulta à Relação Municipal de Medicamentos Essenciais (REMUME) de cada município.

O acesso a este documento foi realizado por meio de consultas aos sítios eletrônicos das secretarias de saúde dos municípios. Quando o acesso à REMUME não foi possível, as informações foram obtidas por contato telefônico.

A fim de garantir a confiabilidade dos dados, foi solicitado um diálogo com os farmacêuticos das secretarias de saúde de cada município, os quais informaram que fitoterápicos estavam padronizados nos municípios, levando em consideração a lista disponível pela Relação Nacional de Medicamentos Essenciais (RENAME).

Quando citado o termo "fitoterápico industrializado" estão compreendidos aqueles produtos que se encontram em uma forma farmacêutica industrializada, considerados medicamentos fitoterápicos e produtos tradicionais fitoterápicos.

Após a realização da coleta de dados, os resultados foram sistematizados, organizados e analisados com o auxílio do programa Microsoft Excel®2007 para Windows.

O projeto de pesquisa foi submetido e aprovado pelo Comitê de Ética da Universidade Federal do Paraná - Setor de Ciências da Saúde, sob parecer de número 1.363.771 e CAAE 49439615.0.0000.0102.

\section{RESULTADOS E DISCUSSÃO}

A Região Metropolitana de Curitiba (RMC) é composta por 29 municípios (QUADRO 1), localizados no Paraná, estado da região sul do Brasil.

Dentre os 29 municípios que compõem a RMC 6 (20,69\%) não realizam a dispensação de fitoterápicos industrializados. São eles: Balsa Nova, Campo Largo, Campo Magro, Curitiba, 
Lapa e Rio Negro.

Dos municípios que optam por não dispensar fitoterápicos industrializados, apenas o município da Lapa possui em andamento a implantação de um projeto para desenvolver o programa denominado de Farmácias Vivas, recebendo recursos financeiros de $\mathrm{R} \$$ 80.000,00 para fins de custeio do desenvolvimento e estruturação da assistência farmacêutica com a inclusão de plantas medicinais e de medicamentos fitoterápicos no SUS.

Os demais 23 municípios (79,31\%) dispensam algum tipo de fitoterápico industrializado em suas unidades de saúde, complementando o elenco de medicamentos da atenção básica à saúde (QUADRO 1).

QUADRO 1 - MUNICÍPIOS DA REGIÃO METROPOLITANA DE CURITIBA QUE DISPENSAM FITOTERÁPICOS INDUSTRIALIZADOS NA ATENÇÃO BÁSICA À SAÚDE.

\begin{tabular}{|c|c|}
\hline Município & Fitoterápico Dispensado \\
\hline Adrianópolis & Isoflavona de Soja, Xarope de Guaco \\
\hline Agudos do Sul & Isoflavona de Soja, Xarope de Guaco e Fitoterápico Composto* \\
\hline Almirante Tamandaré & Isoflavona de Soja, Xarope de Guaco e Espinheira Santa \\
\hline Araucária & Ginkgobiloba \\
\hline Bocaiuva do Sul & Isoflavona de Soja, Xarope de Guaco e Ginkgobiloba \\
\hline Campina Grande do Sul & Isoflavona de Soja e Ginkgobiloba \\
\hline Campo do Tenente & Xarope de Guaco \\
\hline Cerro Azul & Xarope de Guaco \\
\hline Colombo & Isoflavona de Soja, Xarope de Guaco e Espinheira Santa \\
\hline Contenda & Xarope de guaco, Ginkgobiloba, Sominex ${ }^{*}$ \\
\hline Doutor Ulysses & Xarope de Guaco \\
\hline Fazenda Rio Grande & Xarope de Guaco \\
\hline Itaperuçu & Xarope de Guaco \\
\hline Mandirituba & $\begin{array}{c}\text { Espinheira Santa, Xarope de Passiflora incarnata L, Comprimidos de } \\
\text { Passiflora incarnata L, Isoflavona de Soja, Xarope de Guaco, Ginkgobiloba }\end{array}$ \\
\hline Piên & Xarope de Guaco, Isoflavona de Soja, Garra do Diabo \\
\hline Pinhais & Isoflavona de Soja, Xarope de Guaco e Espinheira Santa \\
\hline Piraquara & Xarope de Guaco \\
\hline Quatro Barras & Castanha da Índia, Ginkgobiloba, Xarope de Guaco \\
\hline Quitandinha & Xarope Guaco, Castanha da Índia comprimido, Ginkgobiloba e Sominex* \\
\hline Rio Branco do Sul & Xarope de Guaco \\
\hline São José dos Pinhais & Xarope de Guaco \\
\hline Tijucas do Sul & Isoflavona de Soja \\
\hline Tunas do Paraná & Xarope de Guaco e Espin \\
\hline
\end{tabular}

*Fitoterápico Composto:Valeriana officinalis I., Crataegusoxyacantha L., Passiflora incarnata L.

FONTE: OS AUTORES, (2016). 
Nos municípios da RMC são 8 os fitoterápicos dispensados (TABELA1), destes, alguns não fazem parte da RENAME, que são o Sominex® (Valeriana officinalis L., Crataegus oxyacantha L., Passiflora edulis S.), a Castanha da Índia (Aesculus hippocastanum L.), o Ginkgo biloba, e a Passiflora incarnata L. em xarope e em comprimido. Os demais encontram-se padronizados na relação Nacional de Medicamentos Essenciais (QUADRO 2).

Observando os fitoterápicos disponíveis nos municípios das RMC (TABELA 1), 4 deles se mostram mais frequentes: Xarope de Guaco (87\%), Isoflavona de Soja (48\%), Ginkgo biloba (30\%) e Espinheira Santa (22\%).

Destes apenas o Ginkgo biloba não se encontra padronizado na lista da RENAME, mas faz parte da REMUME para atender a demanda da população.

TABELA 1 - FITOTERÁPICOS INDUSTRIALIZADOS DISPONÍVEIS NAS UNIDADES DE SAÚDE DE MUNICÍPIOS DA RMC.

\begin{tabular}{lccc}
\hline Denominação Genérica & Forma Farmacêutica & Frequência & $\%$ \\
\hline 1 - Passiflora incarnata L. & Comprimido & 1 & 4 \\
1.1 - Passiflora incarnata L. & Xarope & 1 & 4 \\
2 - Garra do Diabo & Cápsula ou comprimido. & 1 & 4 \\
3 - Castanha da índia & Cápsula ou comprimido. & 2 & 8 \\
4 - Fitoterápico Composto* & Comprimido & 3 & 13 \\
5 - Espinheira Santa & Cápsula ou comprimido. & 5 & 22 \\
6 - Ginkgo biloba & Cápsula ou comprimido. & 7 & 30 \\
7 - Isoflavona de Soja & Cápsula ou comprimido. & 11 & 48 \\
8 - Guaco & Xarope & 20 & 87 \\
\hline
\end{tabular}

* Composto de Valeriana officinalis I., Crataegusoxyacantha L., Passiflora incarnata L. FONTE: OS AUTORES, (2016).

Quando se avalia a diversidade dos fitoterápicos industrializados oferecidos nas unidades de saúde, observa-se que, em média, os municípios dispensam 1 ou 3 fitoterápicos (44\% e 39\% respectivamente), e a minoria opta por dispensar 2, 4 ou 6 fitoterápicos ( $9 \%$, 4\% e $4 \%$ respectivamente).

A ampliação da fitoterapia no SUS começou a ocorrer de forma decisiva, após a publicação de duas diretrizes de grande importância na área de plantas medicinais e 
fitoterápicos, que são: a Política Nacional de Plantas Medicinais e Fitoterápicos (PNPMF), que visa a melhoria da atenção à saúde, e a Política Nacional de Práticas Integrativas e Complementares (PNPIC), aprovada pela Portaria $\mathrm{n}^{\circ}$ 971, de 03 de Maio de 2006, que contribuiu com o estímulo da inserção de novos recursos terapêuticos de forma complementar incluindo, além da Fitoterapia, a Homeopatia, a Medicina Tradicional Chinesa/Acupuntura, o Termalismo/Crenoterapia. Posteriormente foi acrescentada a estas a Medicina Antroposófica (BRASIL, 2006a; BRASIL, 2006b; BRASIL, 2006d).

A partir da regulamentação desta prática integrativa, o SUS conseguiu inserir os fitoterápicos na lista dos componentes básicos da assistência farmacêutica, proporcionando um acesso mais fácil da população a estes produtos (BRASIL, 2013).

Desta maneira, o Ministério da Saúde, procurando auxiliar as práticas integrativas e complementares, incluiu alguns medicamentos fitoterápicos e produtos tradicionais fitoterápicos no elenco do componente básico da Assistência Farmacêutica, totalizando atualmente 12 fitoterápicos (QUADRO2), listados na RENAME (BRASIL, 2007; BRASIL, 2009; BRASIL, 2012c).

QUADRO 2 - ULTIMA REVISÃO DOS FITOTERÁPICOS PRESENTES NA RENAME.

\begin{tabular}{|c|c|}
\hline Denominação Genérica & Forma Farmacêutica/ Descrição \\
\hline 1- Alcachofra (Cynarascolymus L.) & Cápsula, comprimido, drágea, solução oral e/ou tintura. \\
\hline 2- Aroeira (SchinusterebinthifoliusRaddi) & Gel e/ou óvulo. \\
\hline 3- Babosa (Aloe vera (L.) Burm. f.) & Creme. \\
\hline 4- Cáscara-sagrada (Rhamnuspurshiana DC.) & Cápsula e/ou tintura. \\
\hline 5- Espinheira-santa (MaytenusofficinalisMabb.) & Cápsula, emulsão, solução oral e/ou tintura. \\
\hline 6- Garra-do-diabo (Harpagophytumprocumbens) & Cápsula ou comprimido. \\
\hline 7- Guaco (MikaniaglomerataSpreng.) & Cápsula, solução oral, tintura e/ou xarope. \\
\hline 8- Hortelã (Mentha x piperita L.) & Cápsula. \\
\hline 9- Isoflavona-de-soja (Glycinemax (L.) Merr.) & Cápsula ou comprimido. \\
\hline 10- Plantago (PlantagoovataForssk.) & Pó para dispersão oral. \\
\hline 11- Salgueiro (Salixalba L.) & Comprimido. \\
\hline $\begin{array}{l}\text { 12- Unha-de-gato (Uncaria tomentosa (Willd. ex } \\
\text { Roem. \&Schult.) DC.) }\end{array}$ & Cápsula, comprimido e/ ou gel. \\
\hline
\end{tabular}

FONTE: RENAME,(2015). 
Na lista da RENAME os fitoterápicos estão no grupo do Componente Básico da Assistência Farmacêutica, disponíveis para que cada município elenque aqueles medicamentos de interesse, a serem relacionados em suas próprias listas (REMUME), bem como disponibilizados para dispensação aos usuários do SUS.

A elaboração da REMUME em cada município acontece por meio de encontros que mobilizam profissionais de diversas áreas, que em conjunto buscam pelos medicamentos que atendam as necessidades epidemiológicas da população naquela região, tendo assim a RENAME como base para elaboração deste elenco (RENAME, 2014).

Estudos demonstram que frequentemente os profissionais envolvidos desconhecem a existência das políticas públicas e diretrizes nacionais que tratam sobre 0 uso de fitoterápicos. O que pode contribuir com a não adesão ou com o pequeno elenco de medicamentos fitoterápicos padronizados nos municípios, uma vez que estes documentos servem de instrumento de orientação principalmente aos gestores, no momento de idealizarem a implantação de novos serviços (ARAÚJO et al., 2014).

Além disso, a resistência ao uso de medicamentos "naturais", aqui verificada por alguns municípios, pode estar relacionada à preferência pelo modelo biomédico, no qual os profissionais, muitas vezes devido a sua formação, acabam desconhecendo ou não acreditando no potencial terapêutico dos fitoterápicos industrializados. Outros fatores podem estar relacionados com a dificuldade de implantar programas de fitoterapia, como o compromisso por parte dos gestores e profissionais bem qualificados que aceitem prescrever fitoterápicos (MARANHÃO, 2011).

Estudos relatam que os profissionais médicos apresentam dificuldades quando se trata da prescrição de fitoterápicos. Sendo necessária uma melhor capacitação destes profissionais, que pode ser iniciada já na graduação, onde as universidades poderiam incluir em seu currículo disciplinas que englobem o assunto fitoterápico (NASCIMENTO et al., 2016).

Além dos municípios aqui estudados, outros Estados como São Paulo, Rio de Janeiro e Ceará já implantaram a fitoterapia no SUS tendo como base as políticas que incentivam esta inclusão (MARANHÃO, 2011; REIS et al., 2004; SILVA et al., 2006)

Segundo o Ministério da Saúde, a disponibilidade destes medicamentos proporciona um desempenho importante em cuidados na atenção básica à saúde, além de auxiliar na ampliação de alternativas de um tratamento seguro, eficaz e bem aceito pelos pacientes (BRASIL, 2012b).

Com a distribuição dos fitoterápicos nas unidades de saúde é estimulado o uso de 
recursos naturais na prevenção de doenças e recuperação da saúde, além de possibilitar a adoção, no SUS, de alternativas complementares de terapia devidamente amparadas por políticas públicas que incentivam a implantação dos fitoterápicos no SUS (BRASIL, 2006b).

De maneira semelhante ao observado neste estudo o xarope de guaco foi o fitoterápico mais disponibilizado para uso no SUS em instalações de saúde e nos municípios do Estado de São Paulo (CACCIA-BAVA et al., 2017).

A maior frequência dos fitoterápicos Xarope de Guaco, Espinheira Santa e Isoflavona de Soja pode estar relacionada com o fato de serem medicamentos disponibilizados pelo Consórcio Paraná Saúde, que tem padronizado estes medicamentos para compra pelos municípios que aderiram a esta forma de aquisição (CONSÓRCIO PARANÁ SAÚDE, s.d).

Além das opções fornecidas pelo consórcio, o município tem a autonomia de adquirir os demais medicamentos por meio de processos licitatórios, o que acontece com aqueles que não estão presentes no elenco pré-estabelecido do Consórcio Paraná Saúde ou que não estão na RENAME atual. A aquisição dos fitoterápicos presentes na RENAME está de acordo com a Portaria n ${ }^{\circ} 1.555$ de 2013, que define os valores e aprova as normas de financiamento na assistência farmacêutica no âmbito do SUS (BRASI, 2013).

Grande parte dos municípios da RMC apresenta um pequeno elenco de fitoterápicos industrializados disponíveis para dispensação na rede de atenção básica, o que demonstra a dificuldade enfrentada pelos municípios em conseguir expandir as opções de produtos fitoterápicos no componente básico, oferecido pelo serviço público.

De modo geral, observamos que a maioria dos municípios opta por dispensar algum tipo de fitoterápico industrializado, porém, o elenco disponibilizado ainda é pequeno, quando comparado a outros municípios que apresentam uma diversidade maior destes produtos em suas unidades de saúde (SILVA et al., 2006).

Estudos demostram que o uso de fitoterápicos se justifica, pois estes apresentam boa eficácia, possuem um menor custo, contribuem com a adesão de pacientes ao tratamento, além de possibilitar aos profissionais de saúde outra opção de tratamento (TOMAZZONI, 2004; LORENZI, MATOS, 2002; FIGUEREDO et al., 2014; CALIXTO, 2001).

\section{CONCLUSÃo}

De acordo com o presente estudo, a maioria dos municípios da $\operatorname{RMC}(79,31 \%)$ apresenta algum tipo de fitoterápico industrializado disponibilizado no elenco de medicametos 
medicamentos da atenção básica à saúde.

O incentivo do uso de fitoterápicos como terapia complementar, melhora o acesso da população a tratamentos integrativos e complementares além de proporcionar o uso sustentável da biodiversidade brasileira, o fortalecimento da agricultura familiar e o desenvolvimento tecnológico e industrial da saúde.

E, embora ocorram várias ações no sentido da implementar os fitoterápicos no SUS, esse crescimento ainda não foi suficiente para torná-los uma prática frequente nos serviços de saúde.

Esta implantação efetiva na atenção básica provavelmente acontecerá quando alguns fatores sejam considerados, como a disponibilidade de recursos, a sensibilização dos gestores em vários níveis, bem como dos demais envolvidos nesta questão.

\section{REFERÊNCIAS}

ARAÚJO, W.R.M. et al. Inserção da fitoterapia em unidades de saúde da família de São Luiz, Maranhão: realidade, desafios e estratégias. Rev. Bras. de Medicina de Família e Comunidade, v.9, n.32, p.258-263, 2014.

BRASIL. Lei no 8.080, de 19 de Setembro de 1990. Dispõe sobre as condições para a promoção, proteção e recuperação da saúde, a organização e o funcionamento dos serviços correspondentes e dá outras providências. Diário Oficial da União, Brasília, DF, 19 set.1990.

BRASIL. Ministério da Saúde. Secretaria de Políticas de Saúde. Proposta de Política Nacional de Plantas Medicinais e Medicamentos Fitoterápicos. Ministério da Saúde, Secretaria de Políticas de Saúde, Departamento de Atenção Básica. Brasília: Ministério da Saúde, 2001.

BRASIL. Ministério da Saúde. Portaria № 971, de 03 de Maio de 2006. Aprova a Política Nacional de Práticas Integrativas e Complementares no SUS. Diário Oficial da União, Brasília, DF, 03 maio. 2006a.

BRASIL. Ministério da Saúde. Política Nacional de Plantas Medicinais e Fitoterápicos. Secretaria de Ciências, Tecnologia e Insumos Estratégicos, Departamento de Assistência Farmacêutica. Série B. Textos Básicos de Saúde. Brasília-DF. 2006b. 
BRASIL. Decreto nํ 5.813, de 22 de Junho de 2006._Aprova a Política Nacional de Plantas Medicinais e Fitoterápicos e dá outras providências. Diário Oficial da União, Brasília, DF, 22 jun. 2006c.

BRASIL. Ministério da Saúde. Portaria № 1.600, de 17 de Julho de 2006. Aprova a constituição do Observatório das Experiências de Medicina Antroposófica no Sistema Único de Saúde (SUS). Diário Oficial da União, Brasília, DF, 17 Jul. 2006d.

BRASIL. Ministério da Saúde. Portaria N³237, de 24 de dezembro de 2007. Aprova as normas de execução e de financiamento da assistência farmacêutica na atenção básica em saúde. Diário Oficial da União, Brasília, DF, 24 dez. 2007.

BRASIL. Ministério da Saúde. Portaria № 2982, de 26 de novembro de 2009. Aprova as normas de execução e de financiamento da assistência farmacêutica na atenção básica. Diário Oficial da União, Brasília, DF, 26 nov. 2009.

BRASIL. Ministério da Saúde. Caderno de Atenção Básica. Práticas Integrativas e Complementares. Plantas Medicinais e Fitoterapia na Atenção Básica. Brasília, DF, 2012a.

BRASIL. Ministério da Saúde. Pesquisa e produção de fitoterápicos ganham reforço. Portal da Saúde, 2012b.

BRASIL. Ministério da Saúde. Portaria № 533 de 28 de março de 2012. Estabelece o elenco de medicamentos e insumos da Relação Nacional de Medicamentos Essenciais (RENAME) no âmbito do Sistema Único de Saúde. Diário Oficial da União, Brasília, DF, 28 mar. 2012c.

BRASIL. Ministério da Saúde. Portaria № 1.555, de 30 de julho de 2013. Dispõe sobre as normas de financiamento e de execução do Componente Básico da Assistência Farmacêutica no âmbito do Sistema Único de Saúde. Diário Oficial da União, Brasília, DF, 30 jul. 2013.

BRASIL. Ministério da Saúde. Agência Nacional de Vigilância Sanitária. Resolução RDC $n^{\circ} 26$ de 13 de maio de 2014. Dispõe sobre o registro de medicamentos fitoterápicos e o registro e a notificação de produtos tradicionais fitoterápicos. Diário Oficial da União, Brasília, 13 mai. 2014. 
CALIXTO, J. B. Medicamentos Fitoterápicos. In: YUNES, R. A.; CALIXTO, J. B. Plantas Medicinais sob a ótica da Química Medicinal Moderna. Santa Catarina: Editora Universitária. p. 297-315. 2001.

CACCIA-BAVA, M. C. G. G.; BeRTONI, B. W.; PEREIRA, M. A. S.; MARTINEZ, E. Z. Disponibilidade de medicamentos fitoterápicos e plantas medicinais nas unidades de atenção básica do Estado de São Paulo: resultados do Programa Nacional de Melhoria do Acesso e da Qualidade da Atenção Básica (PMAQ). Ciência \& Saúde Coletiva 2017; 22(5):1651-1659.

CONSÓRCIO PARANÁ SAÚDE. Histórico, s.d. Disponível em: <http://www.consorcioparana saude.com.br/modelo1. asp?id=1>. Acesso em: 12/01/2016.

FERREIRA, T.S. et al. Phytotherapy: an introduction to its history, use and application. Revista Brasileira de Plantas Medicinais, v.16, n.2, p.290-298, 2014.

FIGUEREDO, C.A. et al. A Política Nacional de Plantas Medicinais e Fitoterápicos: construção, perspectivas e desafios. Physis vol.24 n.2, Rio de Janeiro 2014.

LORENZI, H.; MATOS, F. J. A. Plantas Medicinais no Brasil: nativas e exóticas. Nova Odessa, SP: Instituto Plantarum, 2002.

MARANHÃO, D. Análise Situacional de Seis Programas de Fitoterapia Brasileiros. 49 f. Monografia (Especialização em Gestão da Inovação em Fitomedicamentos) - Complexo Tecnológico de Medicamentos, Fundação Oswaldo Cruz Farmanguinhos, Rio de Janeiro, 2011.

NASCIMENTO JÚNIOR, B.J. et al. Avaliação do conhecimento e percepção dos profissionais da estratégia de saúde da família sobre o uso de plantas medicinais e fitoterapia em Petrolina-PE, Brasil. Revista Brasileira de Plantas Medicinais, v.18, n.1, p. 57-66, 2016.

NIERO, R.; MALHEIROS, A.; BITTENCOURT, C. M. S.; BIAVATTI, M. W.; LEITE, S. N.; CECHINEL FILHO, V. Aspectos Químicos e Biológicos de Plantas Medicinais e Considerações sobre Fitoterápicos. In: BRESOLIN, T. M. B.; CECHINEL FILHOR, V. 
Ciências Farmacêuticas Contribuição ao Desenvolvimento de novos Fármacos e Medicamentos. Itajaí: Univali, p. 11-56. 2003.

REIS, M.C.P. et al. Experiência na implantação do programa de fitoterapia do município do Rio de Janeiro. Divulgação em Saúde para Debate, v. 30, p. 42-49, 2004.

RELAÇÃO NACIONAL DE MEDICAMENTOS ESSENCIAIS: RENAME 2014. Ministério da Saúde, Secretaria de Ciência, Tecnologia e Insumos Estratégicos, Departamento de Assistência Farmacêutica e Insumos Estratégicos. - 9. ed. rev. e atual. - Brasília : Ministério da Saúde, 2015.

SILVA, M.I.G. et al. Utilização de fitoterápicos nas unidades básicas de atenção à saúde da família no município de Maracanaú (CE). Rev. Bras. De Farmacognosia, v.16, n. 4, p. 455462, 2006.

TOMAZZONI, M, I. Subsídios para a Introdução do uso de Fitoterápicos na Rede Básica de Saúde do Município de Cascavel/Pr. Dissertação (Mestrado em Enfermagem) - Setor Ciências da Saúde, Universidade Federal do Paraná, Curitiba, 2004. 